\title{
Sempre, o meritório penhor a quem certifica publicações
}

\author{
Harley E. A. Bicas, Cristina Muccioli, Mauro Goldchmit, Mauro S. O. Campos, Paulo E. Correa Dantas, \\ Samir J. Bechara, Vital Paulino Costa
}

Tradicionalmente, ao começar novo ano, o corpo editorial dos Arquivos Brasileiros de Oftalmologia agradece aos que com ele colaboraram, examinando mais agudamente os artigos encaminhados à publicação. Nesse mister de analistas, comentando a consistência de conteúdo e da apresentação do trabalho e opinando conclusivamente sobre a conveniência de sua aceitação, ou de sua rejeição, essas pessoas sobretudo ajudam os autores. De fato, ao indicarem a necessidade de correções, ou ao sugerirem a prudência de se evitar a exposição pública de escritos que possam depor contra os próprios autores, esses analistas transcendem as funções de julgadores, convertendo-se em professores, conselheiros, amigos (certamente muito mais do que se opinassem pela pura e simples publicação da matéria). Ao colaborarem no aperfeiçoamento de um trabalho tornam-se, nele, freqüentemente, mais importante que certos co-autores cujas participações periféricas merecessem, eventualmente, mais agradecimentos que a inclusão do nome na relação dos que o produziram. É por isso que os Arquivos Brasileiros de Oftalmologia oferecem a esses colaboradores, com atuações sempre mantidas em sigilo, a oportunidade de -- uma vez concluido todo o processo e sido decidida a publicação do trabalho - manifestarem suas identidades, aparecendo publicamente como analistas. Essa, todavia, é uma condição que muitos acham desconfortável, preferindo a manutenção do segredo sobre a autoria das opiniões. Ou, então, uma condição que implica endosso (também público) à forma como o trabalho aparece, o que pode não ser conveniente ao analista (mesmo que o trabalho seja bom). Desse modo, a maioria ainda prefere ficar no anonimato. O que não justifica que se deixe de reconhecer a excelente colaboração prestada.

Mas além dos nomes sempre relacionados em cada número da revista, em sua página de apresentação, muitos outros são acionados pelos Editores para o exercício dessa atividade. Realmente, em 2004 foram recebidos e catalogados pelos A.B.O. 250 trabalhos, o que exige a orientação de cerca de quinhentas revisões. Se apenas os nomes de nossa conhecida lista de conselheiros fossem acionados, caberiam cerca de sete análises a cada um. Convenha-se, uma carga pesada. Daí a necessidade de convites fortuitos (mas que se podem repetir) a pessoas de reconhecidas capacitações profissionais e experiência na redação científica, para complementar o trabalho daqueles conselheiros editoriais.

Seria lastimável que esses abnegados colaboradores, os conselheiros editoriais "ad hoc", deixassem de ser por nós citados (embora esse não lhes tenha sido o motivo da ajuda prestada). Por isso, em nome dos autores, que tanto se beneficiaram pelas pedagógicas análises; em nome dos leitores, cujo aproveitamento pôde ser mais bem cuidado; e em nosso nome, pelo prestígio conferido à revista, os agradecimentos muito sinceros a:

Revisores "Per Review” - 2004

Acácio Alves Souza Lima Filho

Adamo Lui Netto

Adriana dos Santos Forseto

Aisa Haidar Lani

Alexandre Lima de Andrade

Alfredo Tranjan Neto

Amarylis Avakian

Amélia Kamegasawa

Ana Estela Betesti P. Ponce Sant'anna

André Augusto Homsi Jorge

André Barbosa Castelo Branco

André Correa de Oliveira Romano

André Luís Borba da Silva

André Luiz Land Curi

André Marcelo Vieira Gomes

Andrea Araújo Zin

Augusto de Queiróz Duarte

Belquiz Rodrigues do Amaral Nassaralla

Breno Barth Amaral de Andrade

Carlos Alexandre de Amorim Garcia

Carlos Eduardo Leite Arieta

Carlos Fumiaki Uesugui

Carlos Roberto Neulfeld

Carlos Rubens Lucas de Figueiredo

Carlos Teixeira Brandt

Carmo Mandia Jr

Celina Tamaki Monteiro de Castro

César Lipener

Christiane Rolim de Moura

Cláudio Renato Garcia

Clélia Maria Erwenne

Cleusa Coral Ghanem

Consuelo Bueno Diniz Adam

Cristina Maria Bittencourt Garrido

Davi Araf

Denise de Vuono Chinzon

Denise Fornazari de Oliviera

Diane Roussel Marinho

Edison João Geraissate Filho

Edméa Rita Temporini

Edson Procianoy

Eduardo Jorge Carneiro Soares

Eduardo Martines

Eduardo Melani Rocha

Eduardo Minelli 
Eduardo Sone Soriano

Elcio Hideo Sato

Eliana Aparecida Forno

Elisabeto Ribeiro Gonçalves

Emerson Francisco Pereira das Neves

Eric Pinheiro de Andrade

Érika Alessandra G. Silvino Rodrigues

Eurípedes da Mota Moura

Fábio Ejzebaum

Fausto Uno

Fernanda Bom Duarte

Fernando César Abib

Flávio Jaime da Rocha

Flávio Rocha Lima Paranhos

Francisco Eduardo Lopes de Lima

Geraldo de Barros Ribeiro

Helia Soares Angoti

Italo Mundialino Marcon

Jacob Moyses Cohen

João Amaro Ferrari Silva

João Antonio Prata Jr.

João Carlos de Miranda Gonçalves

John Helal Jr.

José Américo Bonatti

José Antonio de Almeida Milani

José Eutrópio S. Vaz Queiróz

José Guilherme de Carvalho Pecego

José Paulo Cabral Vasconcellos

José Ricardo de Abreu Reggi

José Ricardo de Carvalho Lima Rehder

José Vital Filho

José Wilson Cursino

Joyce Hisae Yamamoto

Juliana Maria Ferraz Sallum

Keila Miriam Monteiro de Carvalho

Laurentino Biccas Neto

Leiria de Andrade Neto

Leopoldo Magacho dos Santos Silva

Lúcia Miriam Dumont Lucci

Luciana Lucci

Luciana Peixoto dos Santos

Luciane Chaves Fernandes

Luciene Barbosa de Sousa

Luís Carlos Ferreira de Sá

Luís Eduardo Morato Rebouças de Carvalho

Luiz Alberto Soares Melo Jr.

Luiz Antonio Vieira

Magno Antonio Ferreira

Maira Morales

Marcelo Carvalho da Cunha

Marcelo Francisco Gaal Vadas

Márcia Lowen

Marco Aurélio Lana Peixoto

Marcos Carvalho da Cunha

Marcos Wilson Sampaio

Maria Angélica Dimantas

Maria Auxiliadora M. F. Sibinelli

Maria Cristina Martins

Maria de Lourdes M. M. Villas Boas
Maria Rosa Bet de Moraes e Silva

Marisa Aparecida Polati

Maristela Amaral Palazzi

Marivaldo Castro de Oliveira

Marta Maria Motono Chojniak

Maurício Bastos Pereira

Maurício Della Paolera

Mauro Nishi

Mauro Plut

Mauro Waiswol

Miguel Angelo Gontijo Alvares

Moyses Eduardo Zajdenweber

Nelson Alexandre Sabrosa

Newton Kara-José Jr.

Niro Kasahara

Norma Allemann

Núbia Cristina de Freitas Maia

Patricia Ioschpe Gus

Paulo Afonso Batista dos Santos

Paulo André Polisuk

Paulo César Silva Fontes

Paulo Gelman Vandergorn

Paulo Góis Manso

Paulo Sérgio Moraes Barros

Renato Ambrósio Jr.

Renato Augusto Neves

Renato Giovedi Filho

Ricardo Belfort

Ricardo Morschbacher

Ricardo Suzuki

Ricardo Themudo Lessa Waetge

Roberto Lauande Pimentel

Roberto Pedrosa Galvão Filho

Roberto Pinto Coelho

Rodrigo Jorge

Ronaldo Boaventura Barcellos

Rosa Maria Graziano

Rosalia Maria Simões A. Foschini

Rosane da Cruz Ferreira

Rui Barroso Schimiti

Ruth Miyuki Santo

Saly Moreira

Samuel Rymer

Seiji Hayashi

Sérgio Felberg

Sérgio Vanetti Burnier

Sidney Júlio de Faria e Sousa

Sílvia Prado Smit Kitadai

Simone Finzi

Simone Haber Duellberg Von Faber Bison

Solange Rios Salomão

Teruo Aihara

Tomás Fernando Scalamandré Mendonça

Vera Lúcia Degaspare Monte Mascaro

Walter Yukihiko Takahashi

Wesley Ribeiro Campos

Wilmar Roberto Silvino

Wilson de Freitas

Zélia Maria da Silva Corrêa 